\title{
Differential equations and high-energy expansion of two-loop diagrams in $D$ dimensions *
}

\author{
A.V. Bogdan and R.N. Lee \\ Budker Institute of Nuclear Physics, 630090 Novosibirsk, Russia
}

\begin{abstract}
New method of calculation of master integrals using differential equations and asymptotical expansion is presented. This method leads to the results exact in space-time dimension $D$ having the form of the convergent power series. As an application of this method, we calculate the two-loop master integral for "crossedtriangle" topology which was previously known only up to $O(\epsilon)$ order. The case when a topology contains several master integrals is also considered. We present an algorithm of the term-by-term calculation of the asymptotical expansion in this case and analyze in detail the "crossed-box" topology with three master integrals.
\end{abstract}

\section{Introduction}

High precision of modern high-energy experiments stimulates interest to two-loop calculations. These calculations, in comparison with one-loop calculations, are technically much more complicated. The tensor reduction and integration-by-parts (IBP) method [1,2] effectively reduces the integral, corresponding to any multi-loop diagram, to the linear combination of the master integrals (MIs). The coefficients in this linear combination often have poles of some finite order at $\epsilon=0$ (space-time dimension is $D=4-2 \epsilon)$. Therefore, it is necessary to know the expansion of the master integrals in $\epsilon$, at least, a few first terms of this expansion. Successful method of calculating this expansion term-by-term is based on the application of the differential equations method $[3,4,5,6]$. The results for two-loop diagrams are usually expressed in terms of (generalized) harmonic polylogarithms $[5,6]$ (see, however, Ref. [4]). Due to rapid increase of calculational complexity when increasing the order of expansion, the calculations using this method are typically done up to $O(\epsilon)$ terms. However, it may be not sufficient for the calculation of some diagrams. Therefore, it is highly desirable to calculate master integrals exactly in $D$. One of the most powerful methods of such calculation is the

\footnotetext{
ऋ Work supported by the Russian Fund of Basic Researches, project 03-02-16529-a.

Email addresses: A.V.Bogdan@inp.nsk.su (A.V. Bogdan), R.N.Lee@inp.nsk.su (R.N. Lee).
} 
application of Mellin-Barnes transformation [7,8]. However, for the case of several external invariants and/or many internal lines this method becomes hard to apply.

In this paper, we present a method of obtaining the asymptotic expansion (AE) in inverse powers of some large scale $s$ of multi-loop master integrals exact in space-time dimension $D$. Moreover, this expansion has a finite radius of convergence which can be determined from ODE theory, thus being a power series representation accessible for numerical calculations in definite kinematic region. As an application, we calculate series representations of two-loop diagram presented in Fig.1 in two different kinematics. Our result can be easily expanded in $\epsilon$ and the $O\left(\epsilon^{0}\right)$ term reproduces the known result. We also present the algorithm allowing to calculate, term-by-term, the asymptotical expansion of the diagrams in Fig.3.

Our method can be described as follows. For the calculation of the master integrals of given topology we differentiate them with respect to $s$, and express the result of differentiation (with help of integration by parts $[1,2]$ ) in terms of MIs of the same topology and its subtopologies. Thus, we obtain, in general case, the linear system of first-order ODEs. We assume that the MIs of subtopologies are already known by means of this method or some other. When the given topology has only one MI, $j$, the system is reduced to one equation. The authors of papers [5,6] also used the differential equations method and searched for the solution in the form of $\epsilon$-expansion imposing the boundary condition at some finite point. Unfortunately, the results obtained by the method proposed in Refs. [5,6] quickly become very cumbersome with increase of the order in $\epsilon$. The original point of our approach is the choice of boundary conditions. We impose boundary condition by fixing a definite term of asymptotical expansion of $j$, namely, the term proportional to the main asymptotics of the solution of the homogeneous part of the differential equation, $j_{h}$. In the cases considered in this paper, choice $\epsilon>0$ $(D=4-2 \epsilon)$ allows to fix the boundary condition by calculation of the main asymptotics of $j$. We calculate this asymptotics by means of standard techniques (Feynman parametrization, Mellin-Barnes representation, etc.). Given the asymptotical expansion of inhomogeneous term, of $j_{h}$, and boundary condition, we find the asymptotical expansion of $j$. From the standard theory of ODE it follows that obtained power series has a finite radius of convergence.

Our approach can be also used in the case of system of several equations. In this case, the additional obstacles connected with finding the homogeneous solution arise. However, we can find the AE of the homogeneous solution up to any finite order. The same is true for solution of initial inhomogeneous system.

Thus, using our approach, one can reduce the calculation of the AE (having the form of covergent power series) of master integral in $D$ dimensions to the calculation of its main asymptotics. The radius of convergence can be determined by the methods of ODE theory.

The remainder of the paper is organized as follows. In Section 2, we consider the case of one MI in the topology. We calculate the AE of the integrals corresponding to the vertex diagrams shown in Fig.1. In Section 3, we describe the algorithm which allows to calculate the AE of MIs in the case of system of differential equations. As an example, we consider the crossed-box topology with three MIs depicted in Fig.3. In the Conclusion, we discuss the main results of the present paper. 

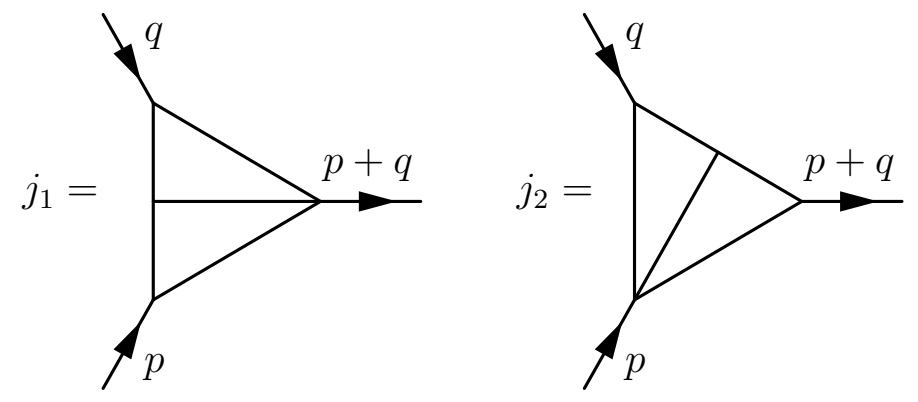

Fig. 1. "Crossed-triangle" topology.

\section{Topology with one master integral}

Consider inhomogeneous differential equation for the MI $j$ with respect to external scale $s$

$$
\frac{d}{d s} j(s)=f(s) j(s)+h(s) .
$$

The solution of this equation is

$$
j(s)=j_{h}(s) \int_{s_{0}}^{s} j_{h}^{-1}\left(s^{\prime}\right) h\left(s^{\prime}\right) d s^{\prime}+j_{h}(s) j_{h}^{-1}\left(s_{0}\right) j\left(s_{0}\right),
$$

where $j_{h}(s)=\exp \left[\int f(s) d s\right]$ is the solution of the corresponding homogeneous equation.

In our approach we are interested in the asymptotical expansion for large $s$ and assume that it is already known for $h(s) j_{h}^{-1}(s)$. Then it is convenient to let $s_{0}$ tend to infinity:

$$
j(s)=\left.j_{h}(s)\left(\int_{s_{0}}^{s} j_{h}^{-1}\left(s^{\prime}\right) h\left(s^{\prime}\right) d s^{\prime}+j_{h}^{-1}\left(s_{0}\right) j\left(s_{0}\right)\right)\right|_{s_{0} \rightarrow \infty} .
$$

For MIs considered in the present paper, the choice $\epsilon>0$ allows to calculate the limit $s_{0} \rightarrow \infty$ separately in the first and the second terms in Eq. (3). In this case we can integrate term-by-term the $\mathrm{AE}$ of the integrand in Eq. (3). In this stage the problem of finding of the whole $\mathrm{AE}$ of $j$ is reduced to the calculation of its main asymptotics. In the more general case, when the limits can not be taken separately, the problem is reduced to the calculation of few first terms of the asymptotics of $j$. As an application of our method, we calculate the AEs of the master integral $j_{1}$ shown in Fig. 1 in two different kinematics. The analytical expression corresponding to the diagram in Fig. 1a has the form

$$
j_{1}\left(s, p^{2}, q^{2}\right)=\frac{1}{(2 \pi)^{2 D}} \int \frac{d^{D} r d^{D} l}{r^{2} l^{2}(r-p)^{2}(l-q)^{2}(r+l)^{2}},
$$

where $s=(p+q)^{2}$ and the usual prescription $r^{2} \rightarrow r^{2}+i 0$ is implied. For convenience, we assume that $p^{2}, q^{2}, s<0$, and the final result should be understood as the analytical continuation from this region. For the kinematics presented in Fig. 1a the differential equation has the form

$$
\frac{\partial j_{1}\left(s, p^{2}, q^{2}\right)}{\partial s}=\frac{(3-D) y}{2 \lambda^{2}} j_{1}\left(s, p^{2}, q^{2}\right)+\frac{1}{2 \lambda^{2}} h\left(s, p^{2}, q^{2}\right),
$$




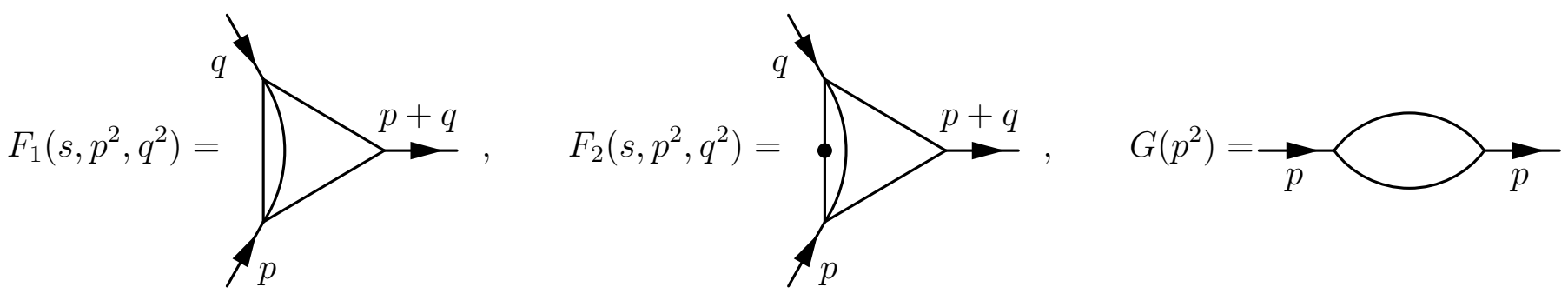

Fig. 2. Master integrals entering Eq.(5).

where

$$
\begin{aligned}
h\left(s, p^{2}, q^{2}\right) & =-\left(y+p^{2}\right) F_{2}\left(q^{2}, p^{2}, s\right)-\left(y+q^{2}\right) F_{2}\left(p^{2}, q^{2}, s\right) \\
& +\frac{(3-D)(3 D-10)}{D-4}\left(F_{1}\left(q^{2}, p^{2}, s\right)+F_{1}\left(p^{2}, q^{2}, s\right)\right) \\
& +\frac{2(3-D)^{2}}{D-4} G\left(p^{2}\right) G\left(q^{2}\right),
\end{aligned}
$$

and $y=(p \cdot q)=\left(s-p^{2}-q^{2}\right) / 2, \lambda^{2}=y^{2}-p^{2} q^{2}$, and $F_{1}\left(p_{1}^{2}, p_{2}^{2}, p_{3}^{2}\right), F_{2}\left(p_{1}^{2}, p_{2}^{2}, p_{3}^{2}\right)$, and $G\left(p_{1}^{2}\right)$ are given by three- and two-point integrals presented in Fig. 2 (dot corresponds to the square of denominator). The asymptotical expansion for $F_{1}$ and $F_{2}$ can be obtained from the well-known formula for off-shell massless triangle with arbitrary powers of denominators [7]. This expansion and formula for $G\left(p_{1}^{2}\right)$ are presented in the Appendix.

The homogeneous solution of Eq. (5) is

$$
j_{1 h}\left(s, p^{2}, q^{2}\right)=(-2 y)^{-1+2 \epsilon}\left(1-\frac{p^{2} q^{2}}{y^{2}}\right)^{-1 / 2+\epsilon} \stackrel{s \rightarrow \infty}{\longrightarrow}(-s)^{-1+2 \epsilon} .
$$

The choice $\epsilon>0$ allows us to take the limit separately in the first and the second terms in Eq. (3). Indeed, the leading asymptotics of $h\left(s, p^{2}, q^{2}\right)$ is $s^{\epsilon}$. Thus, the integrand in Eq. (3) behaves as $s^{-1-\epsilon}$ and the integral converges when its lower limit is replaced by $\infty$. Therefore, we can confine ourselves to calculation of the main asymptotics of $j_{1}$ that determines the limit

$$
\left.\left(j_{1 h}^{-1}\left(s_{0}, p^{2}, q^{2}\right) j_{1}\left(s_{0}, p^{2}, q^{2}\right)\right)\right|_{s_{0} \rightarrow \infty}
$$

We calculate this asymptotics with the help of Feynman parametrization

$$
j_{1}\left(s, p^{2}, q^{2}\right)=\frac{\Gamma[1+2 \epsilon]}{(4 \pi)^{D}} \int_{0}^{\infty} \frac{\prod_{i=1}^{5} d x_{i} \delta\left(1-x_{12345}\right)\left(x_{13} x_{24}+x_{5} x_{1234}\right)^{-1+3 \epsilon}}{\left(-s x_{3} x_{4} x_{5}-p^{2} x_{3}\left(x_{5} x_{12}+x_{1} x_{24}\right)-q^{2} x_{4}\left(x_{5} x_{12}+x_{2} x_{13}\right)\right)^{1+2 \epsilon}} .
$$

where $x_{i_{1}, i_{2}, \ldots}=x_{i_{1}}+x_{i_{2}}+\cdots$. The region, providing the main asymptotics, is

$$
0 \leqslant x_{3} \sim x_{4} \ll x_{1} \sim x_{2} \sim x_{5} \leqslant 1
$$


The result of integration is

$$
j_{1}\left(s, p^{2}, q^{2}\right) \stackrel{s \rightarrow \infty}{\longrightarrow} \frac{\pi^{2}}{(4 \pi)^{D}} \frac{\Gamma[2 \epsilon]}{\epsilon \sin (\pi \epsilon) \sin (2 \pi \epsilon)} \frac{(-s)^{-1+2 \epsilon}}{\left(-q^{2}\right)^{2 \epsilon}\left(-p^{2}\right)^{2 \epsilon}} .
$$

We use the following expansion

$$
\lambda^{-2} j_{1 h}^{-1}\left(s, p^{2}, q^{2}\right)=(-s)^{-1-2 \epsilon} \frac{2 \Gamma[\epsilon]}{\Gamma[2 \epsilon]} \sum_{k, l=0}^{\infty} \Gamma\left[\begin{array}{c}
k+l+1+2 \epsilon, k+l+1+\epsilon \\
k+1+\epsilon, l+1+\epsilon
\end{array}\right] \frac{\left(p^{2} / s\right)^{k}\left(q^{2} / s\right)^{l}}{k ! l !}
$$

where $\Gamma\left[\begin{array}{l}x_{1}, x_{2}, \ldots \\ y_{1}, y_{2}, \ldots\end{array}\right]=\frac{\Gamma\left[x_{1}, x_{2}, \ldots\right]}{\Gamma\left[y_{1}, y_{2}, \ldots\right]}=\frac{\Gamma\left[x_{1}\right] \Gamma\left[x_{2}\right] \ldots}{\Gamma\left[y_{1}\right] \Gamma\left[y_{2}\right] \ldots}$. Substituting (12) in Eq. (3) and integrating over $s$, we obtain

$$
\begin{aligned}
& j_{1}=(-2 y)^{-1+2 \epsilon}\left(1-\frac{p^{2} q^{2}}{y^{2}}\right)^{-1 / 2+\epsilon}(4 \pi)^{-D} \\
& \times\left\{\Gamma[2 \epsilon] \frac{\pi^{2}}{\epsilon \sin (\pi \epsilon) \sin (2 \pi \epsilon)}\left(-q^{2}\right)^{-2 \epsilon}\left(-p^{2}\right)^{-2 \epsilon}\right. \\
& +4 \pi^{2} \cot ^{2}(\pi \epsilon)\left(-q^{2}\right)^{-\epsilon}\left(-p^{2}\right)^{-\epsilon}(-s)^{-2 \epsilon} \Gamma[2 \epsilon] \sum_{k, l=0}^{\infty} \frac{\left(p^{2} / s\right)^{k}\left(q^{2} / s\right)^{l}}{k ! l !} \Gamma\left[\begin{array}{c}
k+l+2 \epsilon, k+l+1+\epsilon \\
1+\epsilon, k+1+\epsilon, l+1+\epsilon
\end{array}\right] \\
& -\frac{\pi^{3}}{2 \Gamma[-3 \epsilon] \sin ^{3}(\pi \epsilon)} \sum_{k, l, m, n=0}^{\infty} \frac{\left(p^{2} / s\right)^{k+m}\left(q^{2} / s\right)^{l+n}}{k ! l ! m ! n !} \Gamma\left[\begin{array}{c}
k+l+1+2 \epsilon, k+l+1+\epsilon \\
1+\epsilon, k+1+\epsilon, l+1+\epsilon
\end{array}\right] \\
& \times\left[\frac{(-s)^{-4 \epsilon}}{k+l+m+n+4 \epsilon}\left(m \Gamma\left[\begin{array}{c}
m+n, m+n+2 \epsilon \\
1+\epsilon+m, 1+2 \epsilon+n
\end{array}\right]+\frac{\delta_{n+m=0}}{3 \epsilon \Gamma[1+\epsilon]}\right)\right. \\
& -\frac{(-s)^{-2 \epsilon}\left(-q^{2}\right)^{-2 \epsilon}}{k+l+m+n+2 \epsilon}\left(m \Gamma\left[\begin{array}{c}
m+n, m+n-2 \epsilon \\
1+\epsilon+m, 1-2 \epsilon+n
\end{array}\right]-\frac{\delta_{n+m=0}}{3 \epsilon \Gamma[1+\epsilon]}\right) \\
& -\frac{(-s)^{-3 \epsilon}\left(-p^{2}\right)^{-\epsilon}}{k+l+m+n+3 \epsilon} \Gamma\left[\begin{array}{c}
m+n+\epsilon, m+n-\epsilon \\
-\epsilon+m, 1+2 \epsilon+n
\end{array}\right] \\
& \left.\left.+\frac{(-s)^{-\epsilon}\left(-p^{2}\right)^{-\epsilon}\left(-q^{2}\right)^{-2 \epsilon}}{k+l+m+n+\epsilon} \Gamma\left[\begin{array}{c}
m+n-\epsilon, m+n-3 \epsilon \\
-\epsilon+m, 1-2 \epsilon+n
\end{array}\right]+\left(\begin{array}{c}
p \leftrightarrow \leftrightarrow \\
m \leftrightarrow \\
\leftrightarrow
\end{array}\right)\right]\right\} .
\end{aligned}
$$

Then we make a shift $k \rightarrow k-m, l \rightarrow l-n$ and change the summation order. The finite sums over 
$m, n$ can be expressed in closed forms. The final result reads

$$
\begin{aligned}
j_{1} & =(-2 y)^{-1+2 \epsilon}\left(1-\frac{p^{2} q^{2}}{y^{2}}\right)^{-1 / 2+\epsilon}(4 \pi)^{-D} \\
& \times\left\{\Gamma[2 \epsilon] \frac{\pi^{2}}{\epsilon \sin (\pi \epsilon) \sin (2 \pi \epsilon)}\left(-q^{2}\right)^{-2 \epsilon}\left(-p^{2}\right)^{-2 \epsilon}\right. \\
& +4 \Gamma[2 \epsilon] \pi^{2} \cot ^{2}(\pi \epsilon)\left(-q^{2}\right)^{-\epsilon}\left(-p^{2}\right)^{-\epsilon}(-s)^{-2 \epsilon} \sum_{k, l=0}^{\infty} \frac{\left(p^{2} / s\right)^{k}\left(q^{2} / s\right)^{l}}{k ! l !} \Gamma\left[\begin{array}{l}
l+k+2 \epsilon, l+k+1+\epsilon \\
1+\epsilon, l+1+\epsilon, k+1+\epsilon
\end{array}\right] \\
& +\epsilon^{2} \Gamma[-\epsilon]^{3} \Gamma[\epsilon] \sum_{k, l=0}^{\infty} \frac{\left(p^{2} / s\right)^{k}\left(q^{2} / s\right)^{l}}{k ! l !} \\
& \times\left\{-(-s)^{-4 \epsilon} \Gamma\left[\begin{array}{c}
k+l+4 \epsilon, k+l+1+2 \epsilon \\
1-3 \epsilon, l+1+4 \epsilon, 1+k+\epsilon]
\end{array} \frac{1}{2 \epsilon}{ }_{3} F_{2}\left[\begin{array}{c}
-k, 3 \epsilon, 2 \epsilon \\
1+l+4 \epsilon, 1+2 \epsilon
\end{array}\right] 1\right]\right. \\
& \left.-(-s)^{-2 \epsilon}\left(-q^{2}\right)^{-2 \epsilon} \Gamma\left[\begin{array}{l}
l+k+1-2 \epsilon, k+l+2 \epsilon \\
1-3 \epsilon, l+1-2 \epsilon, 1+k+\epsilon
\end{array}\right] \frac{1}{2 \epsilon}{ }^{3} F_{2}\left[\begin{array}{c}
-k, 3 \epsilon, 2 \epsilon \\
-l-k+2 \epsilon, 1+2 \epsilon
\end{array}\right] 1\right] \\
& +(-s)^{-3 \epsilon}\left(-p^{2}\right)^{-\epsilon} \Gamma\left[\begin{array}{c}
2 \epsilon, \epsilon, k+l+3 \epsilon, k+l+1+\epsilon \\
1-3 \epsilon, 3 \epsilon, 1+l+2 \epsilon, 1+k+\epsilon
\end{array}\right] \\
& \left.\left.+(-s)^{-\epsilon}\left(-p^{2}\right)^{-\epsilon}\left(-q^{2}\right)^{-2 \epsilon} \Gamma\left[\begin{array}{l}
2 \epsilon, k+l+1-\epsilon, k+l+\epsilon \\
1-\epsilon, 1+l-2 \epsilon, 1+k+\epsilon
\end{array}\right]+\left(\begin{array}{l}
p \leftrightarrow q \\
k \leftrightarrow l
\end{array}\right)\right\}\right\} .
\end{aligned}
$$

As known, $j_{1}$ is finite at $D=4$. It is easy to check that $\epsilon$-poles in individual terms cancel and the finite part reproduce the $\mathrm{AE}$ of the well-known result of [9].

Let us consider the homogeneous system of the differential equations in $s$ for all MIs appearing in the subtopologies of $j_{1}$. The point $s=\infty$ is a regular singular point of this system. Then, from the theory of ODE, it follows that the above expansion has a finite radius of convergence, determined by the closest singularity of the coefficients of this system. In our case, it is located in the point $\lambda^{2}=0$. Thus, the convergence region of Eq. (14) is determined by the condition

$$
|s|>\left|\sqrt{-p^{2}} \pm \sqrt{-q^{2}}\right|^{2} .
$$

Now we pass to the calculation of the asymptotical expansion at large $s$ of master integral $j_{2}\left(s, p^{2}, q^{2}\right)$ corresponding to the diagram shown in Fig. 1b,

$$
j_{2}\left(s, p^{2}, q^{2}\right)=j_{1}\left(p^{2}, s, q^{2}\right) .
$$

The differential equation has the form

$$
\frac{\partial j_{2}\left(s, p^{2}, q^{2}\right)}{\partial s}=\left(\frac{D-4}{s}-\frac{(D-3) y}{2 \lambda^{2}}\right) j_{2}\left(s, p^{2}, q^{2}\right)+\frac{1}{2 \lambda^{2}} h\left(s, p^{2}, q^{2}\right),
$$


where

$$
\begin{aligned}
h\left(s, p^{2}, q^{2}\right) & =p^{2} F_{2}\left(q^{2}, p^{2}, s\right)-\frac{p^{2}}{s}\left(q^{2}+y\right) F_{2}\left(s, p^{2}, q^{2}\right) \\
& +\frac{(3 D-10)(D-3)}{s(D-4)}\left(p^{2}+y\right)\left(F_{1}\left(q^{2}, p^{2}, s\right)+F_{1}\left(s, p^{2}, q^{2}\right)\right) \\
& -2 \frac{(D-3)^{2}}{s(D-4)}\left(p^{2}+y\right) G\left(q^{2}\right) G(s) .
\end{aligned}
$$

The homogeneous solution of Eq. (17) is

$$
j_{2 h}\left(s, p^{2}, q^{2}\right)=(-s)^{-2 \epsilon}(-2 y)^{-1+2 \epsilon}\left(1-\frac{p^{2} q^{2}}{y^{2}}\right)^{-1 / 2+\epsilon} \stackrel{s \rightarrow \infty}{\longrightarrow}(-s)^{-1} .
$$

Similar to the previous case, the choice $\epsilon>0$ allows us to take the limit separately in the first and the second terms in Eq. (3). The calculation of the main asymptotics of $j_{2}\left(s, p^{2}, q^{2}\right)$ is slightly more difficult than that for $j_{1}\left(s, p^{2}, q^{2}\right)$. Using the parametric representation we have

$$
j_{2}\left(s, p^{2}, q^{2}\right)=\frac{\Gamma[1+2 \epsilon]}{(4 \pi)^{D}} \int_{0}^{\infty} \frac{\prod_{i=1}^{5} d x_{i} \delta\left(1-\sum x_{i}\right)\left(x_{13} x_{24}+x_{5} x_{1234}\right)^{-1+3 \epsilon}}{\left(-p^{2} x_{3} x_{4} x_{5}-s x_{3}\left(x_{5} x_{12}+x_{1} x_{24}\right)-q^{2} x_{4}\left(x_{5} x_{12}+x_{2} x_{13}\right)\right)^{1+2 \epsilon}} .
$$

As it is well-known, the sum in the argument of the $\delta$-function in Eq. (20) can run over arbitrary subset of $x_{i}$. We choose it as $\sum x_{i}=x_{1235}$. The region, providing the main asymptotics, is

$$
0 \leqslant x_{3} \ll x_{1} \sim x_{2} \sim x_{4} \sim x_{5} \lesssim 1
$$

After the integration over $x_{3}$ we obtain

$$
j_{2}\left(s, p^{2}, q^{2}\right) \stackrel{s \rightarrow \infty}{\longrightarrow} \frac{\Gamma[2 \epsilon]}{(4 \pi)^{D}} \frac{\left(-q^{2}\right)^{-2 \epsilon}}{-s} \int_{0}^{\infty} \frac{d x_{1} d x_{2} d x_{4} d x_{5} \delta\left(1-x_{125}\right) \beta^{-2 \epsilon} x_{4}^{-2 \epsilon}}{\left(\beta+x_{4} x_{1}\right)\left(\beta+x_{4} x_{15}\right)^{1-3 \epsilon}}
$$

where $\beta=x_{1} x_{2}+x_{2} x_{5}+x_{5} x_{1}$. Now we use the Mellin-Barnes representation

$$
\frac{1}{\left(\beta+x_{4} x_{15}\right)^{1-3 \epsilon}}=\frac{1}{\Gamma[1-3 \epsilon]} \int_{-i \infty+0}^{i \infty+0} \frac{d s}{2 \pi i} \frac{\Gamma[1-3 \epsilon-s]}{\beta^{1-3 \epsilon-s}} \frac{\Gamma[s]}{\left(x_{4} x_{15}\right)^{s}}
$$

and take the integrals over $x_{i}$. The final integration over $s$ can be done by applying the Barnes second lemma (see, e.g., [10]) and results in

$$
j_{2}\left(s, p^{2}, q^{2}\right) \stackrel{s \rightarrow \infty}{\longrightarrow} \frac{1}{(4 \pi)^{D}} \frac{\left(-q^{2}\right)^{-2 \epsilon}}{-s} \frac{\Gamma[1-\epsilon]^{3} \Gamma[2 \epsilon]}{\epsilon(1-2 \epsilon)^{2} \Gamma[1-3 \epsilon]}{ }_{3} F_{2}\left[\begin{array}{c}
1,1,1-\epsilon \\
2-2 \epsilon, 2-2 \epsilon
\end{array} \mid 1\right]
$$


The final result for the $\mathrm{AE}$ of $j_{2}\left(s, p^{2}, q^{2}\right)$, for the diagram depicted in Fig.1b, reads

$$
\begin{aligned}
j_{2}= & \frac{(-2 y)^{-1+2 \epsilon}(-s)^{-2 \epsilon}}{(4 \pi)^{D}}\left(1-\frac{p^{2} q^{2}}{y^{2}}\right)^{-1 / 2+\epsilon}\left\{\left(-q^{2}\right)^{-2 \epsilon} \frac{\Gamma[1-\epsilon]^{3} \Gamma[2 \epsilon]}{\epsilon(1-2 \epsilon)^{2} \Gamma[1-3 \epsilon]}{ }_{3} \mathrm{~F}_{2}\left[\begin{array}{c}
1,1,1-\epsilon \\
2-2 \epsilon, 2-2 \epsilon
\end{array}\right]\right. \\
+ & \frac{\Gamma[\epsilon]}{\Gamma[2 \epsilon]} \sum_{k, l=0}^{\infty}\left(\frac{p^{2}}{s}\right)^{k}\left(\frac{q^{2}}{s}\right)^{l}\left[\left(-q^{2}\right)^{-\epsilon}(-s)^{-\epsilon} \frac{\Gamma[1-\epsilon]^{4} \Gamma[\epsilon]^{2}}{2 \epsilon \Gamma[1-2 \epsilon]^{2}} B_{k, l}\right. \\
+ & \frac{\Gamma[1-\epsilon]^{2}}{2 \epsilon \Gamma[1-3 \epsilon, 1-2 \epsilon]}\left(\left(-q^{2}\right)^{-\epsilon}(-s)^{-\epsilon} C_{k, l}^{(1)}+\left(-p^{2}\right)^{-2 \epsilon}\left(-q^{2}\right)^{-\epsilon}(-s)^{\epsilon} C_{k, l}^{(2)}+\left(-p^{2}\right)^{-2 \epsilon}\left(-q^{2}\right)^{-2 \epsilon}(-s)^{2 \epsilon} C_{k, l}^{(3)}\right. \\
& \left.\left.\left.+(-s)^{-2 \epsilon}\left(C_{k, l}^{(4)}+C_{k, l}^{(5)}\right)+\left(-p^{2}\right)^{-2 \epsilon}\left(C_{k, l}^{(6)}+C_{k, l}^{(7)}\right)+\left(-q^{2}\right)^{-2 \epsilon} C_{k, l}^{(8)}\right)\right]\right\}
\end{aligned}
$$

$$
A_{k, l, m, n}=\frac{(-1)^{n+m}}{n ! m !(k-m) !(l-n) !} \Gamma\left[\begin{array}{c}
k+l-m-n+1+2 \epsilon, k+l-m-n+1+\epsilon \\
k-m+1+\epsilon, l-n+1+\epsilon
\end{array}\right]
$$

$$
B_{k, l}=\frac{1}{k+l+\epsilon}\left(A_{k, l, 1,0} \delta_{k>0}-A_{k, l, 0,1} \delta_{l>0}-A_{k, l, 0,0}\right),
$$

$$
\begin{gathered}
C_{k, l}^{(1)}=\sum_{m=0}^{k} \sum_{n=0}^{l} A_{k, l, m, n} \frac{1-\epsilon}{k+l+\epsilon} \Gamma[m+n-\epsilon, m+n-1+\epsilon, 1-2 \epsilon-m, 1+\epsilon-n]= \\
=\frac{\pi^{2}}{\sin [2 \pi \epsilon] \sin [\pi \epsilon]} \frac{\Gamma[2 \epsilon]}{\Gamma[3 \epsilon]} \frac{2}{(k+l+\epsilon) k ! l !} \Gamma\left[\begin{array}{c}
k+l+3 \epsilon, k+l+1+\epsilon \\
k+2 \epsilon, l+1+\epsilon
\end{array}\right],
\end{gathered}
$$

$$
\begin{aligned}
C_{k, l}^{(2)}= & \sum_{m=0}^{k} \sum_{n=0}^{l} A_{k, l, m, n} \frac{\epsilon-1}{k+l-\epsilon} m \Gamma[m+n-3 \epsilon, m+n-1-\epsilon, 2 \epsilon-m, 1+\epsilon-n]= \\
= & \frac{\pi \Gamma[2 \epsilon, 1-3 \epsilon]}{\sin [2 \pi \epsilon]} \frac{2 k}{(k+l-\epsilon) k ! l !} \Gamma\left[\begin{array}{c}
k+l+\epsilon, k+l+1-\epsilon \\
k+1-2 \epsilon, l+1+\epsilon
\end{array}\right],
\end{aligned}
$$

$$
\begin{aligned}
C_{k, l}^{(3)}= & \sum_{m=0}^{k} \sum_{n=0}^{l} A_{k, l, m, n} \frac{3 m \epsilon-n(1-\epsilon)-6 \epsilon^{2}}{k+l-2 \epsilon} m \Gamma[m+n-2 \epsilon, m+n-1-3 \epsilon, 2 \epsilon-m, 2 \epsilon-n]= \\
& =\frac{\pi \sin [\pi \epsilon]}{\sin ^{2}[2 \pi \epsilon]} \Gamma[2 \epsilon, 1-3 \epsilon] \frac{-2}{(k+l-2 \epsilon) k ! l !} k \Gamma\left[\begin{array}{c}
k+l-\epsilon, k+l+1-2 \epsilon \\
k+1-2 \epsilon, l+1-2 \epsilon
\end{array}\right],
\end{aligned}
$$

$$
\begin{aligned}
C_{k, l}^{(4)}= & \sum_{m=0}^{k} \sum_{n=0}^{l} \frac{A_{k, l, m, n}}{k+l+2 \epsilon}\left((\epsilon-1) n \Gamma[m+n, m+n-1+2 \epsilon, 1-2 \epsilon-m,-\epsilon-n] \delta_{n>0}+\right. \\
& \left.+\Gamma[1-2 \epsilon,-\epsilon, 2 \epsilon] \delta_{m+n=0}\right)= \\
& =\frac{\pi}{\sin [2 \pi \epsilon]} \Gamma\left[\begin{array}{c}
k+l+1+\epsilon, k+l+1+2 \epsilon \\
k+1+\epsilon, l+1+\epsilon
\end{array}\right] \frac{\Gamma[-\epsilon]}{(k+l+2 \epsilon) k ! l !}{ }_{3} \mathrm{~F}_{2}\left[\begin{array}{c}
1,-l, 1-\epsilon \\
1+k+\epsilon, 1+2 \epsilon
\end{array} \mid 1\right],
\end{aligned}
$$




$$
\begin{aligned}
C_{k, l}^{(5)}= & \sum_{m=0}^{k} \sum_{n=0}^{l} A_{k, l, m, n} \frac{n(1-\epsilon)-3 m \epsilon+2 \epsilon(1-\epsilon)}{k+l+2 \epsilon} \Gamma[m+n+2 \epsilon, m+n-1+\epsilon, 1-2 \epsilon-m,-2 \epsilon-n]= \\
= & \frac{\pi^{2}}{\sin ^{2}[2 \pi \epsilon]} \frac{\Gamma[2 \epsilon]}{\Gamma[3 \epsilon]} \frac{2}{(k+l+2 \epsilon) k ! l !} \Gamma\left[\begin{array}{c}
k+l+3 \epsilon, k+l+1+2 \epsilon] \\
k+2 \epsilon, l+1+2 \epsilon
\end{array}\right] \\
C_{k, l}^{(6)}= & \sum_{m=0}^{k} \sum_{n=0}^{l} \frac{A_{k, l, m, n}}{k+l}\left[(1-\epsilon) m n \Gamma[m+n-2 \epsilon, m+n-1,2 \epsilon-m,-\epsilon-n] \delta_{m+n>1}\right. \\
& \left.-m \Gamma[1-2 \epsilon,-\epsilon, 2 \epsilon] \delta_{m+n=1}\right]= \\
& \left.=\frac{\pi}{\sin [2 \pi \epsilon]} \frac{\Gamma[1-\epsilon]}{\epsilon} \frac{-k}{(k+l) k ! l !} \Gamma\left[\begin{array}{c}
k+l+2 \epsilon, k+l+1 \\
k+1, l+1+\epsilon
\end{array}\right]{ }_{3} \mathrm{~F}_{2}\left[\begin{array}{c}
-l, 2 \epsilon, 1-\epsilon \\
k+1,1+2 \epsilon
\end{array}\right] 1\right] \delta_{l+k>0} \\
C_{k, l}^{(7)}= & \sum_{m=0}^{k} \sum_{n=0}^{l} \frac{A_{k, l, m, n}}{k+l}(3 m \epsilon-n(1-\epsilon)-2 \epsilon(1+2 \epsilon)) m \Gamma[m+n, m+n-1-\epsilon, 2 \epsilon-m,-2 \epsilon-n] \delta_{m>0}
\end{aligned}
$$

Again, the convergence region is determined by the condition (15). Similar to the previous case, at $D=4$ we reproduce from Eq. (25) the AE of the well-known result of [9].

\section{Topology with several master integrals}

For the case of several MIs of the same topology, when Eq.(1) turns into the system of ODE, the formal solution of the corresponding homogeneous system is $T \exp \left[\int f(s) d s\right]$. The asymptotical expansion of this solution can be usually found only term-by-term up to some finite order. Our approach can be also applied in this case to find any finite-order term but, usually, not the common term of the AE.

In this Section, we generalize our approach to the case of topology with several master integrals. It worth noting that IBP method always leads to the differential equations for MIs with the coefficients being rational functions of $s$, and the point $s=\infty$ being the regular singular point. Thus, multiplying

the equations by some polynomial, we can reduce them to the following vector form

$$
s P_{1}(1 / s) \frac{\partial \mathbf{J}}{\partial s}+\mathbb{P}_{2}(1 / s) \mathbf{J}=\mathbf{H}
$$




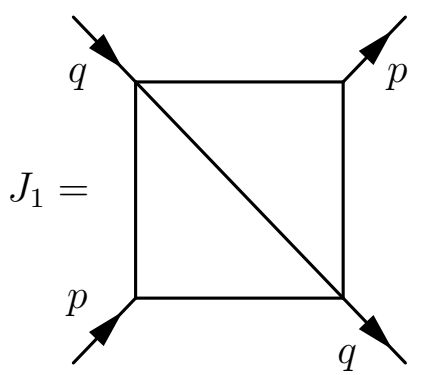

$\mathrm{a}$

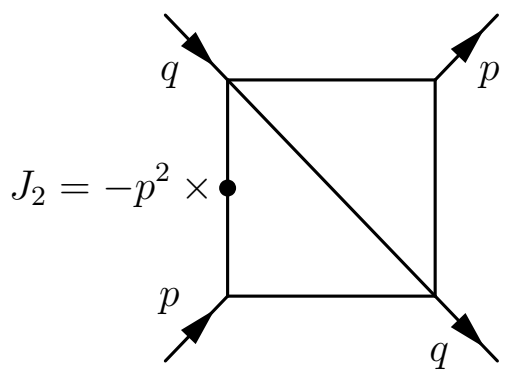

$\mathrm{b}$

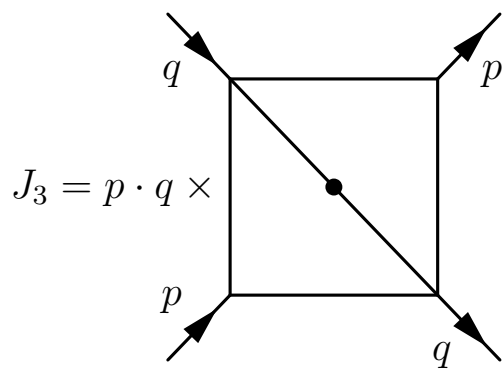

$\mathrm{C}$

Fig. 3. "Crossed-box" topology.

where $P_{1}(x)$ is some polynomial, $P_{1}(0)=1$, and $\mathbb{P}_{2}(x)$ is the polynomial matrix. The algorithm of finding of the AE of $\mathbf{J}$ term-by-term is known from the theory of ODE. Here we will present this algorithm in the case when the asymptotical expansion of the inhomogeneous term is power-like,

$$
\mathbf{H}=\sum_{\alpha \in B} \mathbf{H}(\alpha) s^{-\alpha}
$$

and the following conditions hold

$$
\begin{aligned}
& \text { 1. } \alpha_{i}-\alpha_{j} \text { not integer for } i \neq j, \\
& \text { 2. } \alpha_{i} \notin B,
\end{aligned}
$$

where $\alpha_{i}$ are the eigenvalues of $\mathbb{P}_{2}(0)$. In this case, we can search for the solution of the system (36) in the form of power series

$$
\mathbf{J}=\sum_{\alpha \in A} \mathbf{C}(\alpha) s^{-\alpha},
$$

where $\alpha$ is, in general, not integer, and runs over some discreet set $A$, restricted from below. This set, as well as the coefficients $\mathbf{C}(\alpha)$, are to be determined. It is obvious that $B \subset A$, for convenience we can replace $B \rightarrow A$ and put $\mathbf{H}(\alpha)=0$ if $\alpha \notin B$. Comparing the coefficient in front of equal powers of $s$, we obtain the system of recurrence relations containing $\mathbf{C}(\alpha), \mathbf{C}(\alpha-1), \ldots \mathbf{C}(\alpha-k)$ with some finite, due to the polynomial form of $P_{1}$ and $\mathbb{P}_{2}$, integer $k$

$$
\left[\mathbb{P}_{2}\left(\alpha^{-}\right)-P_{1}\left(\alpha^{-}\right) \alpha\right] \mathbf{C}(\alpha)=\mathbf{H}(\alpha)
$$

where $\alpha^{-}$is the shift operator, $\alpha^{-} f(\alpha)=f(\alpha-1)$. From Eq. (40), we can express $\mathbf{C}(\alpha)$ as a linear combination of $\mathbf{C}(\alpha-1), \ldots \mathbf{C}(\alpha-k)$ and $\mathbf{H}(\alpha)$, unless $\alpha$ coincides with one of the eigenvalues of $\mathbb{P}_{2}(0)$. Therefore, the condition of series breaking from below (at sufficiently small $\alpha$ ) results to $A=B \cup\left\{\alpha_{i}, \alpha_{i}+1, \ldots\right\}$, where $\alpha_{i}$ are the eigenvalues of $\mathbb{P}_{2}(0)$. If $\alpha=\alpha_{i}$, the coefficients $C_{k}(\alpha)$ can not be found from Eq. (40). Due to the conditions (38), this degenerate system remains consistent, and we fix the coefficients $C_{k}(\alpha)$ by using the system and applying the boundary conditions. It is seen, that the boundary conditions are fixed by the terms of AE of $\mathbf{J}$ proportional to $s^{-\alpha_{i}}$.

In the more general case, when there are $\ln s$ in the $\mathrm{AE}$ of $\mathbf{H}$ and/or the conditions (38) do not hold, we have to search the solution in the form of the series (39) with $\mathbf{C}(\alpha)$ replaced by finite sum $\sum_{n} \mathbf{C}^{(n)}(\alpha) \ln ^{n} s$. 
As an example, we consider "crossed-box" topology in forward kinematics (see Fig.3) with three master integrals. In particular, these MIs appear in calculations of radiative corrections to cross sections and of impact factors in QCD. We choose the MIs as shown in Fig.3, i.e.,

$$
\begin{aligned}
& J_{1}=\frac{1}{(2 \pi)^{2 D}} \int \frac{d^{D} r d^{D} l}{r^{2} l^{2}(r+p)^{2}(l+p)^{2}(l-r+q)^{2}}, \\
& J_{2}=-p^{2} \frac{1}{(2 \pi)^{2 D}} \int \frac{d^{D} r d^{D} l}{\left[r^{2}\right]^{2} l^{2}(r+p)^{2}(l+p)^{2}(l-r+q)^{2}}, \\
& J_{3}=(p \cdot q) \frac{1}{(2 \pi)^{2 D}} \int \frac{d^{D} r d^{D} l}{r^{2} l^{2}(r+p)^{2}(l+p)^{2}\left[(l-r+q)^{2}\right]^{2}} .
\end{aligned}
$$

The master integrals (41)-(43) obey the following system of differential equations

$$
\begin{aligned}
\lambda^{2} \frac{\partial J_{1}}{\partial s} & =-2 y \epsilon J_{1}-y J_{2}+\left(q^{2}+y\right) J_{3}+H_{1}, \\
\lambda^{2} u y \frac{\partial J_{2}}{\partial s} & =\epsilon(1-6 \epsilon) y p^{2} \frac{3 u+q^{2}-p^{2}}{4} J_{1}-\frac{y}{2}\left(u y+2\left(p^{4}+4 p^{2}\left(q^{2}-y\right)-q^{2} y\right) \epsilon\right) J_{2} \\
& -\epsilon\left(\lambda^{2} s-y\left(p^{2}-q^{2}\right)\left(u-q^{2}\right)\right) J_{3}+H_{2}, \\
2 \lambda^{2} s u y \frac{\partial J_{3}}{\partial s} & =y p^{2} \epsilon(1-6 \epsilon)\left(\left(p^{2}-2 q^{2}\right)\left(p^{2}-q^{2}\right)-6 \lambda^{2}\right) J_{1} \\
& +2 \epsilon y\left(2 \lambda^{2}\left(4 p^{2}+q^{2}\right)-p^{2}\left(p^{2}-2 q^{2}\right)\left(p^{2}-q^{2}\right)\right) J_{2} \\
& +\left(-\left(p^{2}-q^{2}\right) \epsilon p^{2} y s-4 \lambda^{2} u y \epsilon-2 \lambda^{2} s u \epsilon+\operatorname{suy}\left(3\left(p^{2}+2 y\right) \epsilon-y\right)\right) J_{3}+H_{3},
\end{aligned}
$$

where $u=(p-q)^{2}=2 p^{2}+2 q^{2}-s$ and $H_{i}$ are expressed in terms of MIs of subtopologies of "crossed-box" topology and presented in the Appendix. In this case

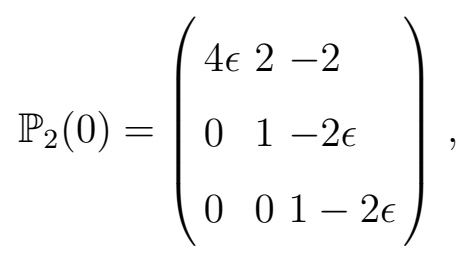

the eigenvalues being $\alpha_{1,2,3}=4 \epsilon, 1,1-2 \epsilon$. Therefore, we have to fix the three terms of $\mathrm{AE}$ of $J_{1}, J_{2}, J_{3}$ proportional to $s^{-4 \epsilon}, s^{-1}, s^{-1+2 \epsilon}$, for example, $C_{1}(4 \epsilon), C_{1}(1), C_{2}(1-2 \epsilon)$.

First, we calculate the asymptotics of $J_{1}$. The Feynman parametrization of $J_{1}$ reads

$$
J_{1}=\frac{\Gamma[1+2 \epsilon]}{(4 \pi)^{D}} \int_{0}^{\infty} \frac{\prod_{i=1}^{5} d x_{i} \delta\left(1-x_{12345}\right)\left(x_{12} x_{34}+x_{5} x_{1234}\right)^{-1+3 \epsilon}}{\left(-p^{2}\left(x_{5} x_{14} x_{23}+x_{2} x_{3} x_{14}+x_{1} x_{4} x_{23}\right)-2 y x_{5}\left(x_{2} x_{4}-x_{1} x_{3}\right)-q^{2} x_{5} x_{12} x_{34}\right)^{1+2 \epsilon}} .
$$

Again, we assume $\epsilon>0$. It is easy to see that there is no contribution $\propto s^{-4 \epsilon}$, therefore, $C_{1}(4 \epsilon)=0$. The main asymptotics $\propto s^{-1}$ is given by the region

$$
0 \leqslant x_{5} \ll x_{1} \sim x_{2} \sim x_{3} \sim x_{4} \leqslant 1
$$


The integral should be treated with care. Assuming that $-p^{2},-q^{2}>0$ and $\operatorname{Im}(s) \neq 0$, we obtain

$$
J_{1} \stackrel{s \rightarrow \infty}{\longrightarrow} \frac{C_{1}(1)}{s}=\frac{i \sigma \pi}{(4 \pi)^{D}} \frac{\left(-p^{2}\right)^{-2 \epsilon}}{s} \frac{\Gamma[\epsilon]^{2} \Gamma[1-2 \epsilon]^{2}}{\Gamma[2-4 \epsilon]}
$$

where $\sigma=\operatorname{sign}(\operatorname{Im} s)=(-i / \pi) \lim _{s \rightarrow \infty} \ln (s / u)$.

Now we calculate the asymptotics of $J_{2}$. When $\epsilon>0$ the integral in Eq. (42) is infrared divergent. This divergence can be eliminated by subtracting from $J_{2}$ the scaleless integral

$$
-p^{2} \frac{1}{(2 \pi)^{2 D}} \int \frac{d^{D} r d^{D} l}{\left(r^{2}\right)^{2} l^{2} p^{2}(l+p)^{2}(l+q)^{2}} .
$$

This subtraction determines the analytical continuation of $J_{2}$ to the region $D<4(\epsilon>0)$. After the Feynman parametrization, we have

$$
\begin{aligned}
J_{2} & =p^{2} \frac{\Gamma[2+2 \epsilon]}{(4 \pi)^{D}} \int_{0}^{\infty} \prod_{i=1}^{5} d x_{i} \delta\left(1-x_{12345}\right) \\
& \times\left[\frac{x_{1}\left(x_{12} x_{34}+x_{5} x_{1234}\right)^{3 \epsilon}}{\left(-p^{2}\left(x_{5} x_{14} x_{23}+x_{2} x_{3} x_{14}+x_{1} x_{4} x_{23}\right)-2 y x_{5}\left(x_{2} x_{4}-x_{1} x_{3}\right)-q^{2} x_{5} x_{12} x_{34}\right)^{2+2 \epsilon}}\right. \\
& \left.-\frac{x_{1}^{-1+\epsilon}\left(x_{345}\right)^{3 \epsilon}}{\left(-p^{2}\left(x_{23} x_{45}+x_{2} x_{3}\right)+2 y x_{3} x_{5}-q^{2} x_{5} x_{34}\right)^{2+2 \epsilon}}\right] .
\end{aligned}
$$

The main asymptotics is given by the region

$$
0 \leqslant x_{2} \sim x_{3} \sim x_{5} \ll x_{1} \sim x_{4} \leqslant 1
$$

The final result reads

$$
J_{2} \stackrel{s \rightarrow \infty}{\longrightarrow} \frac{C_{2}(1-2 \epsilon)}{s^{1-2 \epsilon}}=\frac{\Gamma[2 \epsilon]}{(4 \pi)^{D}} \frac{\pi^{2}\left(-p^{2}\right)^{-2 \epsilon}\left(-q^{2}\right)^{-2 \epsilon}}{\sin (2 \pi \epsilon) \sin (\pi \epsilon)}\left[s^{-1+2 \epsilon}-(-s)^{-1+2 \epsilon}\right] .
$$

The AE of inhomogeneous terms determines the set $B$,

$$
B=\bigcup_{k=1}^{\infty}\{k-\epsilon, k, k+\epsilon, k+2 \epsilon\} .
$$

As we can see, the second condition in Eq. (38) is not satisfied. However, we have checked that the system of recurrence relations remains consistent and, therefore, we can search for the solution in the form (39) with the set $A$ defined as follows

$$
A=\bigcup_{k=1}^{\infty}\{k-2 \epsilon, k-\epsilon, k, k+\epsilon, k+2 \epsilon\}
$$

The set $\{4 \epsilon, 1+4 \epsilon, \ldots\}$ is not included because $C_{1}(4 \epsilon)=0$. To save space, we do not present the explicit form of recurrence relations which are readily obtained from Eqs.(44)-(46). One of the 
consequences of these relations is that $C_{3}(1-2 \epsilon)=C_{2}(1-2 \epsilon)$ and, therefore, the main asymptotics of $J_{3}$ reads

$$
J_{3} \stackrel{s \rightarrow \infty}{\longrightarrow} \frac{\Gamma[2 \epsilon]}{(4 \pi)^{D}} \frac{\pi^{2}\left(-p^{2}\right)^{-2 \epsilon}\left(-q^{2}\right)^{-2 \epsilon}}{\sin (2 \pi \epsilon) \sin (\pi \epsilon)}\left[s^{-1+2 \epsilon}-(-s)^{-1+2 \epsilon}\right] .
$$

\section{Conclusion}

In this paper we present the approach to the calculation of the dimensionally regularized multiloop integrals. This approach is based on the differential equations method and asymptotical expansion. As a demonstration of our method, we have calculated the asymptotical expansions (14) and (25) of the master integral, depicted in Fig.1 in two different kinematical regions. Moreover, we have stressed that these asymptotical expansions have a finite convergence radius determined by Eq.(15), thus being the power series accessible for the numerical calculations. It worth noting that this master integral was known only in $O(\epsilon)$ order so far [6] and this expansion was much more lengthier than our exact representation. Our results can be easily expanded up to any order of $\epsilon$. We have checked that in the limit $\epsilon \rightarrow 0$ our result coincides with the well-known result of Ref. [9]. The result for special kinematics when one or two external momenta are on mass shell $\left(p^{2}=0\right.$ and/or $\left.q^{2}=0\right)$ can be readily obtain from Eqs. (14)-(25). The case when a topology contains several master integrals has also been considered. We present an algorithm of the term-by-term calculation of the asymptotical expansion in this case. The "crossed-box" topology with three master integrals shown in Fig.3 is analyzed in detail.

\section{$5 \quad$ Appendix}

The master integrals appearing in the inhomogeneous parts of differential equations (5), (17), (44)(46) are conveniently expressed via the one-loop off-shell triangle with arbitrary powers of denominators

$$
J(\mu, \nu, \rho)=\frac{1}{(2 \pi)^{D}} \int \frac{d^{D} r}{\left[-r^{2}\right]^{\mu}\left[-(p-r)^{2}\right]^{\nu}\left[-(q+r)^{2}\right]^{\rho}} .
$$

The well-known result [7] for this integral reads

$$
\begin{aligned}
& J(\mu, \nu, \rho)=\frac{i}{(4 \pi)^{D / 2}} \frac{\pi^{2} \csc (D / 2-\mu-\rho) \csc (D / 2-\mu-\nu)}{\Gamma[\mu, \nu, \rho, D-\mu-\nu-\rho]} \sum_{n, m=0}^{\infty} \frac{\left(p^{2} / s\right)^{n}\left(q^{2} / s\right)^{m}}{n ! m !} \times \\
& \left((-s)^{\mu-D / 2}\left(-p^{2}\right)^{D / 2-\mu-\nu}\left(-q^{2}\right)^{D / 2-\mu-\rho} \Gamma\left[\begin{array}{c}
D-\mu-\nu-\rho+n+m, D / 2-\mu+n+m \\
D / 2-\mu-\nu+1+n, D / 2-\mu-\rho+1+m
\end{array}\right]\right. \\
& -(-s)^{-\rho}\left(-p^{2}\right)^{D / 2-\mu-\nu} \Gamma\left[\begin{array}{c}
\rho \\
D / 2-\mu-n+m, D / 2-\nu+n+m
\end{array}\right] \\
& -(-s)^{-\nu}\left(-q^{2}\right)^{D / 2-\mu-\rho} \Gamma\left[\begin{array}{c}
\nu+n+m, D / 2-\rho+n+m \\
\mu+\nu-D / 2+1+n, D / 2-\mu-\rho+1+m
\end{array}\right] \\
& \left.+(-s)^{D / 2-\mu-\nu-\rho} \Gamma\left[\begin{array}{c}
\mu+n+m, \mu+\nu+\rho-D / 2+n+m \\
\mu+\nu-D / 2+n+1, \mu+\rho-D / 2+1+m
\end{array}\right]\right) .
\end{aligned}
$$


Introducing the notation

$$
J(\mu, \nu)=\frac{i}{(4 \pi)^{D / 2}} \frac{\Gamma[\mu+\nu-D / 2, D / 2-\mu, D / 2-\nu]}{\Gamma[\mu, \nu, D-\mu-\nu]},
$$

we obtain for the master integrals appearing in Eqs. (5), (17)

$$
\begin{aligned}
F_{n}\left(s, p^{2}, q^{2}\right) & =(-1)^{n+1} J(1, n) J(1+n-D / 2,1,1), \\
F_{n}\left(p^{2}, q^{2}, s\right) & =(-1)^{n+1} J(1, n) J(1,1,1+n-D / 2), \\
F_{n}\left(q^{2}, p^{2}, s\right) & =(-1)^{n+1} J(1, n) J(1,1+n-D / 2,1), \\
G(s) & =J(1,1)(-s)^{D / 2-2} .
\end{aligned}
$$

The inhomogeneous part of the system (44)-(45) is expressed as follows

$$
\begin{gathered}
H_{1}=\left(q^{2}+y\right) T_{7}-\left(q^{2}+2 y\right) T_{8}, \\
H_{2}=\left(q^{2}-p^{2}-u\right) y(1-2 \epsilon)^{2} T_{1} / 4+\left(q^{2}-p^{2}+u\right) y(1-3 \epsilon)(2-3 \epsilon)(1-2 \epsilon) T_{2} /\left(4 q^{2} \epsilon\right) \\
-y(1-3 \epsilon)(2-3 \epsilon)(1-2 \epsilon) T_{3} /(2 \epsilon)+\left(q^{2}-p^{2}+u\right) y(1-3 \epsilon)(1-2 \epsilon) T_{5} / 4 \\
\quad+\left(p^{2}-q^{2}\right) y(1-3 \epsilon)(1-2 \epsilon) T_{6} / 2+\left(q^{4}-p^{4}+u\left(4 p^{2}-q^{2}+3 y\right) / 2\right) \epsilon y T_{7} \\
\quad-\left(3\left(q^{2}-p^{2} p^{2}+\left(7 p^{2}+q^{2}\right) u+4 u y\right) \epsilon y T_{8} / 2\right. \\
\quad \\
H_{3}=\left(q^{4}-p^{4}-s u\right) y(1-2 \epsilon)^{2} T_{1} / 2-\left(q^{4}-p^{4}+s u\right) y(1-3 \epsilon)(2-3 \epsilon)(1-2 \epsilon) T_{2} /\left(q^{2} \epsilon\right) \\
+s y(1-3 \epsilon)(2-3 \epsilon)(1-2 \epsilon)\left(s T_{3}+u T_{4}\right) / \epsilon+\left(p^{4}-q^{4}+s u\right) y(1-3 \epsilon)(1-2 \epsilon)\left(T_{5}+T_{6}\right) \\
+s y\left(q^{4}-p^{4}+\left(3 p^{2}-q^{2}\right) u+2 u y\right) \epsilon T_{7} \\
-y\left(s u\left(9 p^{2}+4 q^{2}+2 y\right)-\left(p^{4}-q^{4}\right)\left(5 p^{2}-4 q^{2}+2 y\right)\right) \epsilon T_{8},
\end{gathered}
$$

where

$$
\begin{array}{ll}
T_{1}=G^{2}\left(p^{2}\right), & T_{5}=F_{1}\left(p^{2}, q^{2}, u\right), \\
T_{2}=J(1,1) J(1,2-D / 2)\left(-q^{2}\right)^{D-3}, & T_{6}=F_{1}\left(p^{2}, q^{2}, s\right), \\
T_{3}=J(1,1) J(1,2-D / 2)\left(-u^{2}\right)^{D-3}, & T_{7}=F_{2}\left(p^{2}, q^{2}, s\right), \\
T_{4}=J(1,1) J(1,2-D / 2)\left(-s^{2}\right)^{D-3}, & T_{8}=F_{2}\left(p^{2}, q^{2}, u\right) .
\end{array}
$$

\section{References}

[1] K.G. Chetyrkin, A.L. Kataev, and F.T. Tkachev, Nucl. Phys. B 174 (1980) 345;

K.G. Chetyrkin and F.T. Tkachev, Nucl. Phys. B 1192 (1981) 159;

[2] S. Laporta, Int. J. Mod. Phys. A 15 (2000) 5087 arXiv:hep-ph/0102033; 
[3] A.V. Kotikov, Phys. Lett. B 254 (1991) 158;

A.V. Kotikov, Phys. Lett. B 259 (1991) 314;

A.V. Kotikov, Phys. Lett. B 267 (1991) 123;

Z. Bern, L.J. Dixon, and D.A. Kosower, Nucl. Phys. B 412 (1994) 751, arXiv:hep-ph/9306240;

E. Remiddi, Nuovo Cim. A 110 (1997) 1435, arXiv:hep-th/9711188;

R. Bonciani, Acta Phys. Polon. B 30, 3463 (1999);

[4] T. Gehrmann and E. Remiddi, Nucl. Phys. B 580, 485 (2000) arXiv:hep-ph/9912329;

[5] T. Gehrmann and E. Remiddi, Nucl. Phys. B 601, 248 (2001) arXiv:hep-ph/0008287;

T. Gehrmann and E. Remiddi, Nucl. Phys. B 601, 287 (2001) arXiv:hep-ph/0101124;

R. Bonciani, P. Mastrolia and E. Remiddi, Nucl. Phys. B 661, 289 (2003) [Erratum-ibid. B 702, 359 (2004)] arXiv:hep-ph/0301170.

[6] T.G. Birthwright, E.W.N. Glover, and P, Marquard, JHEP 0409 (2004) 042, arXiv:hep-ph/0407343;

[7] E.E. Boos and A.I. Davydychev, Teor. Mat. Fiz. 89 (1991) 56,

[8] S. Friot, D. Greyat, and E. de Rafael arXiv:hep-ph/0505038

[9] N. I. Usyukina and A. I. Davydychev, Phys. Lett. B 332, 159 (1994) arXiv:hep-ph/9402223.

[10] L.J. Slater, "Generalized hypergeometric functions", Cambridge University Press, 1966. 